\title{
APONTAMENTOS DE TEMAS GEOMÉTRICOS PARA ATIVIDADES-HISTÓRICAS-COM-TECNOLOGIAS
}

\section{APPOINTMENTS OF GEOMETRIC THEMES FOR HISTORICAL-ACTIVITIES- WITH-TECHNOLOGIES}

\author{
Anna Beatriz de Andrade Gomes ${ }^{1}$; Giselle Costa Sousa ${ }^{2}$
}

\begin{abstract}
RESUMO
O presente artigo é fruto de uma pesquisa de Iniciação Científica (IC) em andamento, vinculada ao projeto Conexões potenciais entre História da Matemática e TDIC: Aporte para fomento de atividades-históricas-com-tecnologias (PROPESQ/UFRN), fundamentando-se em pesquisadores que tratam do uso da História da Matemática (HM) no ensino, das Tecnologias Digitais (TD) e a Educação Matemática, da Investigação Matemática (IM) e ainda da aliança entre HM, TD e IM. Neste trabalho temos por objetivo geral fazer apontamentos de temas geométricos via produção de planilhas/quadros que alimentarão uma linha do tempo interativa. Esperamos assim, continuar o fomento de atividades-históricas-com-tecnologias, que são atividades que trabalham com problemas históricos auxiliados por tecnologiais digitais via investigação. O foco no campo geométrico se justifica pelo fato do parâmetro tema histórico (obtido pela investigação de edições anteriores deste projeto de IC), que configura a aliança supracitada, aponta para a Geometria como assunto recorrente em tais atividades. Com este propósito, foi realizada uma pesquisa qualitativa de cunho bibliográfico baseando-se no site Mactutor History of Mathematics e complementando com livros de História da Matemática e História das Ciências. Em tal pesquisa procuramos produções de natureza geométrica ao longo da história destacando o nome do estudioso, período em que viveu, nome das principais obras conhecidas e onde é possível encontrá-las, seja online ou físico. Como resultado, tais informações foram organizadas em planilhas que serão expostas, no presente texto, por meio de quadros resumidos, considerando as limitações deste documento, e também são divididas nos seguintes períodos: Idade Antiga, Idade Média e Idade Moderna. Contudo, as planilhas podem ser acessadas na íntegra por meio de um link apresentado que dá acesso a todo levantamento. Além disso, como desdobramento, almeja-se produzir uma linha do tempo interativa online que permita o leitor acessar facilmente todas as informações presentes nas planilhas sobre o personagem histórico desejado e sua produção incluindo acesso/informação de documento que pode servir de base para produção de atividades-históricas-com-tecnologia.
\end{abstract}

Palavras-chave: História da Matemática; Tecnologias Digitais de Informação Comunicação; Investigação Matemática; Atividades-históricas-com-tecnologias; Tema Geométrico.

\footnotetext{
${ }^{1}$ Graduação em andamento pela Universidade Federal do Rio Grande do Norte (UFRN). Natal, RN, Brasil. Endereço para correspondência: Terceira Travessa da Floresta, 09, Nossa Senhora da Apresentação, Natal, RN, Brasil, CEP: 59114-003. E-mail: gomesbeatriz.anna@mail.com.

ORCID iD: https://orcid.org/0000-0002-7642-4526.

${ }^{2}$ Doutora. Universidade Federal do Rio Grande do Norte (UFRN). Professora associada do DMAT (UFRN), Natal, RB, Brasil. Endereço para correspondência: Cirilo Moreira, 261 - Nazaré, Natal, RB, Brasil, CEP: 59062-130. E-mail: gisellematufrn@ mail.com.
}

(D) ORCID iD: https://orcid.org/0000-0003-0213-4179. 


\begin{abstract}
This article is the result of an ongoing Scientific Initiation (IC) research, linked to the project Potential connections between the History of Mathematics and TDIC: Support for the promotion of historical activities with technologies (PROPESQ / UFRN), based on researcher which deal with the use of the History of Mathematics (HM) in teaching, of Digital Technologies (TD) and Mathematical Education, of Mathematical Research (IM) and also of the alliance between HM, TD and IM. In this work we have as a general objective to make notes of geometric themes via the production of spreadsheets / charts that will feed an interactive timeline. Thus, we hope to continue the promotion of historical-activities-with-tecnologies, which are activities that work with historical problems aided by digital technologies through research. The focus on the geometrical field is justified by the fact of the historical parameter (obtained by investigating previous editions of this CI project), which sets up the aforementioned alliance, points to Geometry as a recurring subject in such activities. For this purpose, a research was carried out qualitative bibliography based on the Mactutor History of Mathematics website and complemented with books on the History of Mathematics and History of Science. In such research we look for productions of a geometric nature throughout history, highlighting the name of the scholar, period in which they lived, name of the main works needed and where it is possible to find them, whether online or physical. As a result, such information was organized in spreadsheets that will be exposed, in the present text, by means of summarized tables, considering the limitations of this document, and are also divided in the following periods: Ancient Age, Middle Ages and Modern Age. However, the spreadsheets can be accessed in full through a link presented that gives access to the entire survey. In addition, as an offshoot, we aim to produce an interactive online timeline that allows the reader to easily access all the information present in the spreadsheets about the desired historical character and his production including access / document information that can serve as a basis for the production of historical-activities-with-technologies.
\end{abstract}

Keywords: History of Mathematics; Digital Information and Communication Technologies; Mathematical Investigation; Historical-with-technology-activities; Geometric themes. 


\section{Introdução}

O projeto Conexões potenciais entre História da Matemática e TDIC: Aporte para fomento de atividades-históricas-com-tecnologias (PROPESQ/UFRN) tem por finalidade estudar a aliança entre História da Matemática (HM) e Tecnologias Digitais de Informação e Comunicação (TDIC), via Investigação Matemática, baseando-se nas pesquisas de Sousa (2020a; 2020b) para a aliança e respaldando-se em Miguel e Miorim (2008) quanto a HM usada em sala de aula, Borba e Penteado (2007) para a inclusão de tecnologias digitais no ensino da matemática, e Ponte, Brocado e Oliveira (2009) sobre a Investigação Matemática no ensino.

Nas edições anteriores deste projeto, foram feitos levantamentos em Anais de eventos de pesquisas que apresentassem a aliança ente HM e TDIC. Segundo Costa e Sousa (2017), após este levantamento, notou-se a existência de trabalhos do mesmo viés, entretanto, poucos estudos sobre tal aliança. Além disso, no intuito de dar o aporte inicial para fomento de atividades que trabalham com HM e TDIC, as autoras definiram alguns parâmetros, baseando-se nos trabalhos catalogados em sua pesquisa, que aparecem com mais frequência. São eles os parâmetros: metodologia, tema, software e cunho educacional. Destes, os mais frequentes nos trabalhos foram a metodologia qualitativa, o tema Geometria, o software GeoGebra e o cunho educacional, produtos educacionais do tipo sequência didática.

Posteriomente, afim de continuar o aporte para fomento destas atividades e melhor compreender a proposta da aliança entre HM e TDIC por meio da IM, foi feita uma análise em dissertações que pesquisam a proposta da aliança orientados pela professora Giselle Costa de Sousa entre 2011 a 2019. Apartir deste levantamento (banco de teses e dissertações da Capes), Sousa e Gomes (2020) definiram as atividades-históricas-comtecnologia como sendo atividades que trabalham problemas históricos apoiando-se nas tecnologias por meio da IM. Além disso, também o referido estudo identificou os elementos essênciais que estruturam este tipo de atividade, sendo eles: Elementos prétextuais, Informações básicas, Desenvolvimento e Avaliação.

O presente trabalho é resultado parcial de uma pesquisa de Iniciação Científica (IC) vinculada ao projeto citado anteriormente. Nosso objetivo foi fazer apontamentos de temas históricos por meio de tópicos de História da Matemática voltados para Geometria, considerando o parâmetro tema apresentado por Costa e Sousa (2017), e produzimos 
quadros que apontam potenciais produções que podem suscitar a elaboração de atividades referentes a aliança entre HM e TDIC.

Os quadros foram divididos por período e apresentam informações, baseando-se na linha do tempo do MacTutor History of Mathematics ${ }^{3}$, como nome do estudioso em geometria, período em que viveu, uma breve informação sobre o seu trabalho, título de suas obras e o link de acesso a esse documento, quando disponível. Além disso, com as informações presentes nos quadros, pretende-se produzir uma linha do tempo interativa por meio do site Timetoast Timelines ${ }^{4}$ e fazer um ensaio de uma sequência didática que proponha a aliança entre HM e TDIC. Ressalvamos que esta pesquisa apresenta resultados parciais de um projeto de Iniciação Científica, visto que é um trabalho em andamento, portanto, os quadros não estão concluídos ainda.

Considerando a proposta posta, apresentamos a seguir a metodologia utilizada.

\section{Percurso Metológico}

Para a produção dos quadros, foi utilizada a metodologia qualitativa do tipo bibliográfica. Sobre a pesquisa qualitativa, Garnica (1997) apresenta em seu artigo Algumas notas sobre pesquisas qualitativa e fenomenologia que é muito utilizada quando se trata de resultados e informações sem a importância da quantidade que:

[...] nas abordagens qualitativas, o termo pesquisa ganha novo significado, passando a ser concebido como uma trajetória circular em torno do que se deseja compreender, não se preocupando única e/ou aprioristicamente com princípios, leis e generalizações, mas voltando o olhar à qualidade, aos elementos que sejam significativos para o observador-investigador. (GARNICA, 1997, p. 111, grifo do autor)

Ou seja, a pesquisa qualitativa prima pela qualidade das informações presentes indo além da análise numérica e quantitativa. Por meio de uma pesquisa qualitativa, buscamos reunir uma seleção de informações a partir da análise de documentos.

O estudo de textos e documentos classifica esta pesquisa como do tipo bibliográfica, que tem o objetivo de:

a) proporcionar um aprendizado sobre uma determinada área do conhecimento; b) facilitar a identificação e seleção dos métodos e técnicas a serem utilizados pelo pesquisador; c) oferecer subsídios para a redação da introdução e revisão

\footnotetext{
${ }^{3}$ Link de Acesso: https://bit.ly/3qwRQzT

${ }^{4}$ Link de Acesso: https://bit.ly/2M6mb9G
} 
da literatura e redação da discussão do trabalho científico. (PIZZANI et al, 2012, p. 54).

Logo, esse tipo de pesquisa nos auxilia quanto ao tratamento de dados e nos oferece aporte para a seleção das informações consideradas mais relevantes para o desenvolvimentro da pesquisa proporcionando aprendizado da área e facilitando o apontamento de métodos e técnicas que podem ser adotados e dar subsídios para a fomento de trabalhos voltados para a aliança entre HM, TDIC e IM,.

Inicialmente, utilizamos o site do MacTutor History of Mathematics (O'CONNOR; ROBERTSON, 2019), que apresentava uma linha do tempo visual dos estudiosos na área da matemática e suas respectivas biografias. Durante o encaminhamento desta pesquisa, o site passou por uma atualização, deixando de ter o modelo de linha mais visual e ficando a opção de acesso cronológico das biografias dos personagens históricos. Diante deste fato, entramos em contato com os responsáveis e conseguimos uma cópia do modelo utilizado anteriormente.

Portanto, o objeto de pesquisa utilizado é a cópia do site anterior juntamente com a informações das biografias presentes no site atual e em livros de História da Matemática e História das Ciências, que foram usados como complementos, acrescentando e confrontando informações ao estudo, visto que muitos estudiosos da matemática também foram pesquisadores de muitas outras áreas, como engenharia, astronomia, física, e entre outros.

Quanto aos referidos livros de História da Matemática e História das Ciências, utilizamos fontes como: História da Matemática, do Carl B. Boyer (2012); História da Matemática: Uma visão crítica, desfazendo mitos e lendas, da Tatiana Roque (2012); História da Matemática e suas (re)construções conceituais, do Fumikazu Saito (2015), A Concise History of Mathematics, do D. J. Struik (1954), Lecciones de Historia de las Matemáticas, de Hans Wussing (1998), Companion Encyclopedia of the History and Philosophy of the Mathematical Sciences, de Ivor Grattan-Guinnes (1994), a coleção de livros História da Ciências, de Carlos Augusto Rosa (2012), e entre outros.

Após o levantamento bibliográfico, as informações foram organizadas em planilhas e estão alimentando a linha do tempo. Para a produção da linha do tempo interativa, foi utilizado o site Timetoast Timelines, que permite a visualização da mesma de maneira extensa e permite que o usuário considere os personagens históricos que trabalharam com geometria da época desejada e que também clique no personagem de 
sua preferência e apresente uma imagem ou pintura (quando possível) e um pequeno texto histórico que faça referência ao mesmo. Além disso, que acesse documentos em que suas produções de natureza geométrica se encontram.

Já visto o caminho trilhado por esta pesquisa, exibimos a seguir os resultados obtidos até então.

\section{Resultados e discussões concernentes ao estudo realizado}

Como posto anteriormente, para a produção dos quadros iniciamos baseando-se na linha do tempo do Mactutor, que é separada nos seguintes períodos: Grego, Árabe, Séc. 16 e 17, Séc. 18, início do Séc. 19, fim do Séc. 19 e Séc. 20. Os quadros com a seleção dos tópicos de HM em Geometria são separados por nós, até o momento, não na divisão do Mactutor, mas nos períodos classificados na História Geral como: Idade Antiga, Idade Média e Idade Moderna. Por ser uma pesquisa que está em andamento, os quadros de todos os períodos ainda não foram concluídos e, portanto, temos nesse artigo, os dados coletados até a metade da Idade Moderna.

Compreendemos como Idade Antiga a época entre 800 a.E.C.(antes da Era Comum) e 474 d.E.C.(depois da Era Comum). Para esse período, o representante inicialmente registrado na linha, que trabalhou com geometria , é Baudhayam (800 a.E.C) e encerramos os registros com Antêmio de Tralles (474 d.E.C. - 534 d.E.C.).

Segundo Vázquez, Rey e Boubée (2008), a Idade Média tem início com a queda de Roma, em 476 e finaliza em 1453, com a queda de Constantinopla, logo, o quadro referente a Idade Média seleciona inicialmente o matemático Aryabatha (476-550) e termina com o Leonardo da Vincii (1452-1519).

O registro da Idade Moderna inicia com Scipione del Ferro (1465-1526) e, até o momento, termina com Honoré Fabri (1608-1688). Vale salientar que temos consciência que as possibilidades de nomes e trabalhos nos referidos períodos, aqui apresentada, não se esgota com este levantamento. Além disso, fizemos escolhas para indicar um inicial e final de cada período, mas que podem haver outros anteriores e posteriores, sobretudo, a depender de novos parâmetros de escolhas.

Considerando as limitações previstas na formatação deste documento, a forma que encontramos de tratamento de resultados foi selecionar alguns personagens presentes na 
planilha e dispô-los em pequenos quadros, separados por épocas e sem o tópico histórico referente ao mesmo. Entretanto, a planilha completa é disponibilizada por meio do $\operatorname{link}{ }^{5}$.

Cada quadro apresenta a época que se refere, o período em que o personagem histórico viveu, o nome do personagem, nome de sua(s) obra(s) e onde elas podem ser encontradas, caso estejam disponíveis. O critério de seleção utilizado para os estudiosos presentes neste artigo foram as intenções da produção de um ensaio de produto educacional ou para uma dissertação de mestrado e/ou doutorado que proponha a aliança entre HM e TDIC e na proposta do não-acontecimental ${ }^{6}$, apresentada por Saito (2015). Ressalvamos que em alguns casos, não encontramos registros do nome das obras ou onde elas podem ser enocntradas, porém, tentamos reunir o máximo de informação dado o tempo da pesquisa.

O quadro 1 é intitulado de Recortes do quadro da Idade Antiga e apresenta quatro estudiosos da época da Idade Antiga.

Quadro 1 - Recortes do quadro da Idade Antiga

\begin{tabular}{|c|c|c|c|}
\hline PERÍODO & NOME & OBRAS & DISPONIBILIDADE \\
\hline $\begin{array}{c}\text { 569 A.E.C. }- \\
\text { 475 A.E.C. }\end{array}$ & $\begin{array}{c}\text { Pitágoras de } \\
\text { Samos }\end{array}$ & $\begin{array}{c}\text { Não teve obras de geometria } \\
\text { publicadas }\end{array}$ & - \\
\hline $\begin{array}{c}\text { 460 A.E.C. - } \\
\text { 370 A.E.C. }\end{array}$ & $\begin{array}{c}\text { Demócrito de } \\
\text { Abdera }\end{array}$ & $\begin{array}{c}\text { On numbers, On geometry, } \\
\text { On tangencies, On } \\
\text { mappings, On irrationals } \\
\text { (data desconhecida) }\end{array}$ & Não encontrado. \\
\hline $\begin{array}{c}\text { 287 A.E.C. - } \\
\text { 212 A.E.C. }\end{array}$ & $\begin{array}{c}\text { Arquimedes } \\
\text { de Siracusa }\end{array}$ & $\begin{array}{c}\text { Archimēdous } \text { Panta } \\
\text { sōzomena (data } \\
\text { desconhecida) }\end{array}$ & $\begin{array}{c}\text { Versão traduzida e } \\
\text { comentada: } \\
\text { https://bit.ly/2TjJxcc }\end{array}$ \\
\hline 90 A.E.C. - & $\begin{array}{c}\text { Teodósio de } \\
\text { Bitínia }\end{array}$ & Sphaerics (Séc. 2 A.E.C.) & $\begin{array}{c}\text { Cópia disponível para } \\
\text { compra a versão } \\
\text { bilingue (Árabe e } \\
\text { Latim) pela editora } \\
\text { Franz Steiner Verlag } \\
\text { Wiesbaden GmbH. }\end{array}$ \\
\hline
\end{tabular}

Fonte: Elaborado pelas autoras (2021)

\footnotetext{
${ }^{5}$ Link de acesso as planilhas: https://bit.ly/368ejf0 cujas informações alimentam a linha do tempo

${ }^{6}$ Em linhas gerais o não-acontecimental está relacionado ao que ainda não se mostrou evidenciado passando a ser acontecimento quando a emergência da verdade é acionada por evidências e traz a sua história à tona. De modo simplista poderíamos mencionar que o não-acontecimental não apareceu na história ainda, não teve seu registro historiográfico, ao menos sob determinado ponto de vista. A acontecimentalização ocorreria quando se dá vez e voz aos fatos evidenciados.
} 
O primeiro personagem histórico apresentado no quadro é Pitágoras de Samos (569 a.E.C-475 a.E.C), a quem é atribuído o Teorema de Pitágoras, que se refere a um triângulo retângulo em que o quadrado da hipotenusa é igual a soma dos quadrados dos catetos e, além disso, estudou sobre os cinco sólidos regulares e álgebra geométrica. $\mathrm{O}$ segundo é Demócrtio de Abdera (460 a.E.C. - 370 a.E.C.), que afirmou que o volume de um cone é um terço do cilindro com a mesma base e altura e que o volume de uma pirâmide é um terço do de um prisma com mesma base e altura. Sua obra é conhecida como On numbers, On geometry, On tangencies, On mappings, On irrationals (data desconhecida) e ela não foi encontrada até o momento.

O terceiro estudioso é Arquimedes de Siracusa (287 a.E.C.-212 a.E.C.). Ele estudou a relação entre uma esfera e um cilindro circunscrito de mesma altura e diâmetro e a quadratura de uma parábola. Sua obra é conhecida como Archimēdous Panta sōzomena (data desconhecida). Já o quarto e último matemático do quadro é Teodósio de Bitínia (160 a.E.C-90 a.E.C.). Teodósio trabalhou com geometria da esfera e sua obra, conhecida como Sphaerics (Séc. 2 a.E.C.), tinha a intenção de dar base matemática para estudar astronomia e dar teoremas que generalizem os dados fornecidos por Euclides em os Elementos.

O quadro 2, Recortes do quadro da Idade Média, apresenta os seguintes personagens:

Quadro 2 - Recortes do quadro da Idade Média

\begin{tabular}{|c|c|c|c|}
\hline PERÍODO & NOME & OBRAS & DISPONIBILIDADE \\
\hline $800-873$ & $\begin{array}{c}\text { Jafar } \\
\text { Muhammad } \\
\text { Banu Musa }\end{array}$ & $\begin{array}{c}\text { Premises of the book of } \\
\text { conics (data } \\
\text { desconhecida) }\end{array}$ & Não encontrado. \\
\hline $800-870$ & Mahavira & $\begin{array}{c}\text { Ganita Sara Sangraha } \\
(850)\end{array}$ & $\begin{array}{c}\text { versão com comentários em } \\
\text { inglês: } \\
\text { https://bit.ly/2TkygIE }\end{array}$ \\
\hline $965-1040$ & $\begin{array}{l}\text { Abu Ali al- } \\
\text { Hasan ibn } \\
\text { al-Haytham }\end{array}$ & $\begin{array}{c}\text { Maqālah mustaqsāt fī al- } \\
\text { ashkāl al-hilāliyyah (data } \\
\text { desconhecida) } \\
\text { Maqālah fī anna al-kurah } \\
\text { awsa' al-ashkāl al- } \\
\text { mujassamah allatī } \\
\text { iḥāțatuhā mutasāwiyyah } \\
\text { wa-anna al-dā'irah awsa } \\
\text { al-ashkāl al-musatțahạah } \\
\text { allatī iḥāțatuhā } \\
\text { mutasāwiyyah (data } \\
\text { desconhecida) }\end{array}$ & 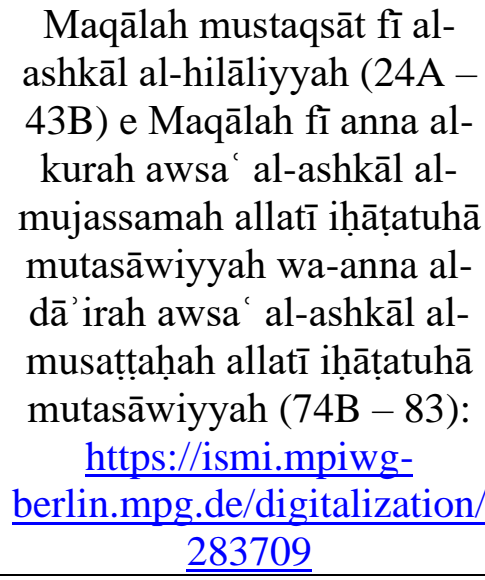 \\
\hline
\end{tabular}


Anna Beatriz de Andrade Gomes e Giselle Costa Sousa

Apontamentos de temas geométricos para Atividades-Históricas-com-Tecnologias

\begin{tabular}{|c|c|c|c|}
\hline $1444-1544$ & $\begin{array}{c}\text { Nilakantha } \\
\text { Somayaji }\end{array}$ & $\begin{array}{c}\text { Tantrasamgraha } \\
(1501)\end{array}$ & $\begin{array}{c}\text { Cópia disponível para } \\
\text { compra na versão traduzida } \\
\text { para inglês pela editora } \\
\text { Book on Demand Ltd. }\end{array}$ \\
\hline
\end{tabular}

Fonte: Elaborado pelas autoras (2021)

No segundo quadro, o primeiro estudioso apresentado é Jafar Muhammad Banu Musa (800-873) que trabalhou com cônicas e contribuiu para textos de geometria. Em sua obra, fez uma revisão crítica sobre Conics, de Apolônio. Seu trabalho se chama Premises of the book of conics (data desconhecida). O segundo personagem inserido no quadro é Mahavira (800-870). Mahavira apresentou processos para calcular o volume de uma esfera e para calcular a raiz do cubo de um número e sua obra de Geometria se chama Ganita Sara Sangral (850).

Em seguida, apresentamos Abu Ali al-Hasan ibn al-Haytham (965-1040). AlHaytham trabalhou a aplicação da geometria na astronomia, onde considerou as áreas da lua como dois círculos que se interceptam. Além disso, estudou sobre a quadratura do círculo. Alguns de seus trabalhos em geometria intitulam-se Maqālah mustaqsāt fì alashkāl al-hilāliyyah (data desconhecida) e Maqālah fì anna al-kurah awsa'al-ashkāl almujassamah allatī iḥātatuhā mutasāwiyyah wa-anna al-dāirah awsa' al-ashkāl almusațțahah allatī iḥâțatuhā mutasāwiyyah (data desconhecida). Finalizando o quadro 2, temos Nilakantha Somayaji (1444-1544), que obteve uma expressão aproximada para um arco da circunferência de um círculo derivando uma expansão de série e depois considerando o limite. Sua obra intitula-se Tantrasamgraha (1501).

Neste segundo quadro, nota-se uma grande predominância de matemáticos islâmicos. Por este motivo, a maioria das obras localizadas encontram-se na sua língua de origem, portanto, levamos em consideração o que estudiosos da área e que conhecem a língua selecionaram como obras de Geometria para apresentá-los nos quadros.

O quadro 3, e último dos apresentado aqui, é o Recortes do quadro da Idade Moderna.

Quadro 3 - Recortes do quadro da Idade Moderna

\begin{tabular}{|c|c|c|c|}
\hline PERÍODO & NOME & OBRAS & DISPONIBILIDADE \\
\hline $\begin{array}{c}1468- \\
1522\end{array}$ & $\begin{array}{l}\text { Johann } \\
\text { Werner }\end{array}$ & $\begin{array}{c}\text { De triangulis sphaericis } \\
\text { libri quatuor; De } \\
\text { meteoroscopiis libri sex } \\
\text { (1907) }\end{array}$ & $\frac{\text { https://archive.org/details/ioa }}{\underline{\text { nnisvernerid00rhgoog }}}$ \\
\hline
\end{tabular}


Anna Beatriz de Andrade Gomes e Giselle Costa Sousa

Apontamentos de temas geométricos para Atividades-Históricas-com-Tecnologias

\begin{tabular}{|c|c|c|c|}
\hline $\begin{array}{c}1508- \\
1555\end{array}$ & $\begin{array}{c}\text { Regnier } \\
\text { Gemma } \\
\text { Frisius }\end{array}$ & $\begin{array}{c}\text { Usus annuli } \\
\text { astronomici (1548) }\end{array}$ & $\underline{\text { https://bit.ly/3obZyPn }}$ \\
\hline $\begin{array}{c}\text { 1584- } \\
1638\end{array}$ & Pierre & $\begin{array}{c}\text { La Construction, } \\
\text { l'usage, } \text { et les } \\
\text { propriétés du quadrant } \\
\text { nouveau de } \\
\text { mathématiques }(1631)\end{array}$ & $\begin{array}{c}\text { Cópia disponível para compra } \\
\text { pela editora Kessinger } \\
\text { Publishing. }\end{array}$ \\
\hline $1597-1652$ & $\begin{array}{c}\text { Jean Charles } \\
\text { de La Faille }\end{array}$ & $\begin{array}{c}\text { gravitatis partium } \\
\text { circulis et ellipsis } \\
\text { (1632) }\end{array}$ & $\begin{array}{c}\text { Cópia disponível na } \\
\text { Informação e Cultura. }\end{array}$ \\
\hline
\end{tabular}

Fonte: Elaborado pelas autoras (2021)

O primeiro a ser apresentado no terceiro quadro é Johann Werner (1468-1522) que trabalhou com geometria esférica e secções cônicas. Seu trabalho só foi publicado 400 anos depois do seu falecimento e intitula-se De triangulis sphaericis libri quatuor; De meteoroscopiis libri sex (1907). O segundo estudioso é Regnier Gemma Frisius (15081555). Regnier usou aplicações trigonométricas para resolver problemas astronômicos. Seu trabalho é conhecido como Usus annuli astronomici (1548).

A seguir, encontramos Pierre Vernier (1584-1638). Em sua obra, conhecida como La Construction, l'usage, et les propriétés du quadrant nouveau de mathématiques (1631), Vernier apresenta uma tabela de senos e um método para derivar os ângulos de um triângulo se seus lados forem conhecidos. Ele também descreve sua invenção mais famosa, a do compasso de calibre vernier, um instrumento para medir o comprimento com precisão. Por fim, tem-se Jean Charles de La Faille (1597-1652). La Faille provou que os centros de gravidade de um setor de um círculo, de uma figura regular nele inscrita, de um segmento de um círculo ou de uma elipse, se encontram no diâmetro da figura. Sua obra se chama Theoremata de centro gravitatis partium circulis et ellipsis (1632).

Ao fazer a análise geral deste terceiro quadro, percebe-se que há um acesso maior às obras que os outros. Consideramos que isso se deve ao fato de serem personagens históricos mais recentes, além de que, as técnicas e os materiais de registro são melhores do que nos períodos anteriores, menos perecíveis, por exemplo.

De posse dos apontamentos de temas geométricos para atividades-históricas-comtecnologias as informações foram postas em nossa linha do tempo interativa com o recurso do site Timetoast. O Timetoast é um site que permite a produção de linhas do tempo, sendo possível a criação de eventos e espaços de tempo. Para a divisão das épocas, 
utilizamos a opção espaços de tempo, pois visualmente, ele cria uma linha temporal, representada por uma linha roxa, que mostra seu início e fim.

Para os personagens, utilizamos a opção eventos. Nela, aparece a data de nascimento, nome do personagem e uma imagem ou pintura referente a ele (caso tenha). Ao clicar no personagem, aparece um pequeno tópico com informações sobre suas contribuições na geometria. Inclui-se aqui, por exemplo, informações de suas obras, ou ainda, quando disponível, o link para acessá-las. Vale salientar que como é uma plataforma em inglês, os períodos que aparecerem com BCE (Before the Common Era) ao lado, são os anos antes da Era Comum. Mostramos um exemplo na Figura 1:

Figura 1 - Linha do tempo interativa

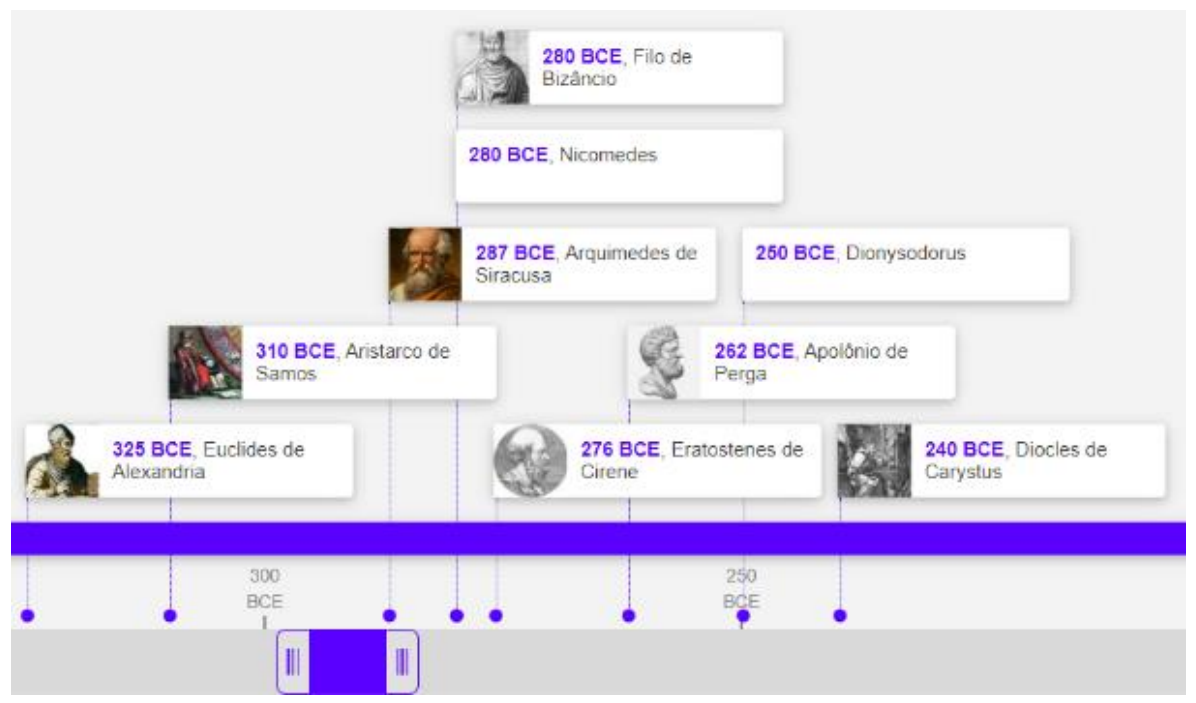

Fonte: Print do site Timetoast (2021)

A linha do tempo interativa, intitulada Matemáticos em Geometria, foi produzida e alimentada com todas as informações presentes na planilha fruto de nossa pesquisa de apontamentos para temas geométricos de atividades na ótica da aliança. Assim como os quadros, a linha do tempo ainda não está concluída. Porém, ela já pode ser acessada por este $\operatorname{link}{ }^{7}$. No site, também há a opção de como quem está utilizando o site prefere ver a linha, Timeline, que são todos os personagens dispostos em uma grande linha (como mostrado na Figura 1), ou em List, que seriam os personagens mostrados em forma de lista já com imagem e tópico histórico.

\section{${ }^{7}$ Matemáticos em Geometria timeline | Timetoast timelines}


Além da alimentação das planilhas/quadros e da linha do tempo interativa com mais informações levantadas, também almejamos fazer um ensaio de uma sequência didática, considerando o parâmetro de produto educacional apresentado por Costa e Sousa (2017), selecionando um dos personagens históricos catalogados no levantamento e sua respectiva obra.

\section{Considerações finais}

Este artigo é o desdobramento de pesquisas anteriores de iniciação científica vinculadas ao projeto Conexões potenciais entre História da Matemática e TDIC: aporte para fomento de atividades-históricas-com-tecnologias. O objetivo desse trabalho é fazer a seleção de tópicos de HM/obras que fazem referências a contribuições em Geometria para fomentar produções que proponham a aliança entre História da Matemática e TDIC via IM. Tendo realizado levantamento em temas potenciais, obtemos como resultado planilhas informativas com apontamentos de tópicos a serem explorados na aliança. Como resultado, além dos quadros sintéticos aqui expostos, tais dados têm sido organizados em uma linha do tempo com auxílio do site Timetoast.

Desse modo, para a produção de atividades-históricas-com-tecnologias, encaminhamos que se faça uma seleção de tema histórico e, como ponto de partida, aconselha-se a análise dos quadros aqui apresentados. Além disso, se recomenda que seja seguida a estruturação de uma atividade com essa característica, norteando-se pelos elementos essenciais indicados por edição anterior dessa pesquisa de IC, a saber: Elementos pré-textuais, Informações Básicas, Desenvolvimento e Avaliação. Desse modo, consideramos fomentar a produção de produtos educacionais na ótica da aliança entre HM, TDIC e IM.

\section{Referências}

BOYER, Carl B.; MERZBACH, Uta C.. História da Matemática. 3. ed. Brasil: Blucher, 2012.

BORBA, Marcelo de Carvalho; PENTEADO, Miriam Godoy. Informática e Educação Matemática. 3 ed. Belo Horizonte: Autêntica, 2007.

COSTA, Allyson Emanuel Januário da; SOUSA, Giselle Costa de. Investigando a Conjunção entre História da Matemática e Tecnologias de Informação e Comunicação, por meio de um Levantamento Bibliográfico em Eventos 
Internacionais de Educação Matemática. Revista Boletim Cearense de Educação e História da Matemática, Ceará, v.04, n.11, 06 - 21. (2017).

GARNICA, Antonio Vicente Marafioti. Algumas notas sobre Pesquisa Qualitativa e Fenomenologia. Interface - Comunicação, Saúde, Educação, v.1, n.1, 1997.

GRATTAN-GUINNES, Ivor. (ed.). Companion Encyclopedia of the History and Philosophy of the Mathematical Sciences. London \& New York: Routledge. 1994.

MIGUEL, Antonio; MIORIM, Maria Ângela. História na Educação Matemática: propostas e desafios. - 1 ed., 2 reimp. - Belo Horizonte: Autêntica, 2008.

PIZZANI, Luciana; SILVA, Rosemary Cristina da; BELLO, Suzelei Faria; HAYASHI, Maria Cristina Piumbato Innocentini. A arte da pesquisa bibliográfica na busca do conhecimento. Revista Digital de Biblioteconomia e Ciência da Informaçã, v.10, n.1, p53-66, 2012.

PONTE, João Pedro da; BROCARDO, Joana; OLIVEIRA, Hélia. Investigações

Matemáticas na Sala de Aula. - 2. Ed. - Belo Horizonte: Autêntica Editora, 2009.

O'CONNOR, John J.; ROBERTSON, Edmundo F.. Mactutor History of Mathematics archive. Disponível em: <https://www-history.mcs.st-and.ac.uk/>. Acesso em: 02 nov. 2019.

ROQUE, Tatiana Martins. História da Matemática: Uma visão crítica, desfazendo mitos e lendas. Brasil: Zahar, 2012.

ROSA, Carlos Augusto. História da Ciências: a ciência e o triunfo do pensamento científico no mundo contemporâneo. 2. ed. Brasília: Fundação Alexandre de Gusmão, 2012.

ROSA, Carlos Augusto. História da Ciências: da antiguidade ao renascimento científico. 2. ed. Brasília: Fundação Alexandre de Gusmão, 2012.

ROSA, Carlos Augusto. História da Ciências: pensamento científico e a ciência no século XIX. 2. ed. Brasília: Fundação Alexandre de Gusmão, 2012.

SAITO, Fumikazu. História da Matemática e suas (re)construções conceituais. São Paulo: Livraria da Física, 2015.

SOUSA, Giselle Costa de. Aliança entre HM, TDIC e IM: fundamentos e aplicações. In: REMATEC: Revista de Matemática, Ensino e Cultura, Ano 15, Fluxo Contínuo, 2020a, p.117-136. Disponível em:

<http://www.rematec.net.br/index.php/rematec/article/view/239>. Acesso em: 27 ago. 2020 .

SOUSA, Giselle Costa de. Aliança entre História da Matemática e Tecnologias via Investigação Matemática: reflexões e práticas. São Paulo: Editora Livraria da Física, 2020 b. 
SOUSA, Giselle Costa De; GOMES, Anna Beatriz De Andrade. Aporte para a promoção de atividades-históricas-com-tecnologia. Research, Society and Development, v. 9, n. 5, p. 1689-1699, 2020.

STRUIK, Dirk J. A Concise History Of Mathematics. 4a ed. London. Bell and Sons, Inc., 1954.

VÁZQUEZ, P. S.; REY, G.; BOUBÉE, C.. El concepto de función a través de la Historia. Revista Ibero americana de Educação Matemática. v. 4, n. 6, pp. 141- 151, Dez. 2008.

WUSSING, Hans. Lecciones de Historia de las Matemáticas. 1998. 